\title{
Pharmacological Validation of the Prepulse Inhibition of Startle Response in Larval Zebrafish using a Commercial Automated System and Software
}

\author{
Nancy Saana Banono ${ }^{1,2}$, Camila V. Esguerra ${ }^{1,2}$ \\ ${ }^{1}$ Chemical Neuroscience Group, Centre for Molecular Medicine Norway (NCMM), Faculty of Medicine, University of Oslo ${ }^{2}$ Section for Pharmacology and \\ Pharmaceutical Biosciences, Department of Pharmacy, Faculty of Mathematics and Natural Sciences, University of Oslo
}

\section{Corresponding Author}

Camila V. Esguerra

c.v.esguerra@ncmm.uio.no

\section{Citation}

Banono, N.S.,

Esguerra, C.V. Pharmacological

Validation of the Prepulse Inhibition of

Startle Response in Larval Zebrafish

using a Commercial Automated System

and Software. J. Vis. Exp. (161), e61423,

doi:10.3791/61423 (2020).

\section{Date Published}

July 2,2020

DOI

$10.3791 / 61423$

URL

jove.com/video/61423

\section{Abstract}

While there is an abundance of commercial and standardized automated systems and software for performing the prepulse inhibition (PPI) assay in rodents, to the best of our knowledge, all PPI assays performed in the zebrafish have, until now, been done using custom made systems which were only available to individual groups. This has thereby presented challenges, particularly with regard to issues of data reproducibility and standardization. In the present work, we generated a protocol that utilizes commercially available automated systems to pharmacologically validate the PPI assay in larval zebrafish. Consistent with published findings, we were able to replicate the results of apomorphine, haloperidol and ketamine on the PPI response of 6 days post-fertilization zebrafish larvae.

\section{Introduction}

The zebrafish (Danio rerio) larva is a suitable candidate for modelling psychiatric diseases such as schizophrenia (reviewed by Gawel et al. ${ }^{1}$ ) because of the numerous advantages it possesses. These include a fully sequenced genome with $70 \%$ sequence homology to human orthologues ${ }^{2}$, existence of forward and reverse genetic tools to manipulate the genome and to identify the contribution of a given gene towards development or disease ${ }^{3}$, and the presence of major human/rodent neurotransmitters in the zebrafish brain ${ }^{4}$. There is an availability of several neuro-phenotypic domains in zebrafish, such as anxiety, learning and memory ${ }^{3}$. Optical transparency and sensitivity to the major classes of neurotropic drugs makes it an ideal 
candidate of choice for pharmacological manipulations and phenotypic drug screening 5,6 .

To perform high throughput drug screening, automation and the presence of a robust endophenotype is highly important $^{7}$. For instance, a variety of automatic recording techniques have been developed for measuring larval zebrafish behavior such as thigmotaxis, startle response, optokinetic response, optomotor response, habituation, prey capture, sleep/wake behavior, locomotor behavior and several others ${ }^{6}$. While some laboratories develop custombuilt systems for automated measurements and analysis of some of these behaviors, there are commercially available imaging and software systems $8,9,10,11$. Prepulse inhibition (PPI), a form of sensorimotor gating in which the startle response is reduced when a weak non-startling stimulus is presented briefly before the startling stimulus, has been used as an endophenotype for studying schizophrenia in animal models (reviewed by ${ }^{12,13}$ ). In addition, acoustic startle response (ASR) and $\mathrm{PPI}$ have played useful roles in studying hearing and auditory function in animal models including the zebrafish $^{14,15}$. The larval zebrafish displays a characteristic C-start in response to an unexpected startling stimulus that is lessened by a weaker stimulus called the prepulse. The C-start has long been described as an escape behavior controlled by distinct neural cell populations and has been thoroughly characterized in the larval zebrafish ${ }^{15}, 16,17$.

There is an abundance of commercial and standardized automated systems and software for performing the PPI assay in rodents ${ }^{18,19,20}$. However, to the best of our knowledge, all the PPI assays performed in the zebrafish until now have been done using custom made systems which are only available to the individual groups $15,16,21,22$. This presents challenges for achieving data reproducibility and replicability with regard to standardization ${ }^{23}$.

Recently, a known vendor in the zebrafish community developed a set-up embedded with a fast camera and PPI generator add-ons to carry out the PPI assay in larval zebrafish $^{24}$. The camera records at 1000 frames per second which enables the recording of fast acting behaviors such as the C-start, while the PPI generator allows for user-controlled delivery of various acoustic stimuli to evoke a startle response $^{24}$. Here, we combine the aforementioned system with a commercially available comprehensive software package designed for the automated analysis of complex behaviors $^{11}$, to generate a protocol for performing PPI response assays in larval zebrafish. We pharmacologically validate the PPI response using 1) apomorphine, a dopamine agonist known to cause deficits in PPI; 2) haloperidol, a dopamine antagonist and antipsychotic known to enhance PPI and 3) ketamine, a NMDA receptor antagonist known to modulate PPI.

\section{Protocol}

All animal experiments were approved by the Norwegian Food Safety Authority experimental animal administration's supervisory and application system (FOTS-18/106800-1).

\section{Zebrafish husbandry}

1. Set up matings of wild type adult male and female zebrafish (Danio rerio) stocks, maintained under standard conditions $^{25}$ the evening before. Here, Tupfel long-fin (TL) strain is used.

2. Remove barriers the next morning and allow to mate through natural spawning.

3. Collect eggs out of the mating tanks. 
4. Remove unfertilized eggs and other debris, then transfer eggs to petri dishes $(n=60)$ and raise in an incubator at $28{ }^{\circ} \mathrm{C}$ in embryo medium: $1.5 \mathrm{mM}$ HEPES, $\mathrm{pH} 7.6,17.4$ $\mathrm{mM} \mathrm{NaCl}, 0.21 \mathrm{mM} \mathrm{KCl}, 0.12 \mathrm{mM} \mathrm{MgSO} 4$, and $0.18 \mathrm{mM}$ $\mathrm{Ca}(\mathrm{NO} 3)_{2}$.

5. Renew half of the embryo medium and remove dead larvae daily until $6 \mathrm{dpf}$.

NOTE: All experiments were performed on individual larvae at 6 days post-fertilization (dpf).

\section{Pharmacological agents and pre-treatment of larvae}

1. Dissolve apomorphine and ketamine in E3 medium to make $500 \mu \mathrm{M}$ and $10 \mathrm{mM}$ stock solutions respectively.

2. Dissolve haloperidol in $100 \%$ dimethyl sulfoxide (DMSO) to make a $10 \mathrm{mM}$ stock solution. The final concentration of DMSO used was $0.1 \%$.

3. Use $0.1 \%$ DMSO and E3 medium as vehicle controls.

4. Use the following final concentrations of drugs: 10 $\mathrm{mg} / \mathrm{mL}$ of apomorphine, $1 \mathrm{mM}$ ketamine and $20 \mu \mathrm{M}$ haloperidol $^{16}$.

5. Pre-expose the apomorphine and ketamine groups larvae for $10 \mathrm{~min}$ and the haloperidol and DMSO vehicle control groups for $20 \min ^{16}$.

\section{Setup prior to the behavior test}

1. On the day of the experiment, transfer larvae and all relevant materials into the experiment room. Set the experiment room to a temperature of $27 \pm 1{ }^{\circ} \mathrm{C}$.

2. Ensure that the background noise in the test chamber is as low as possible, preferably not more than $45 \mathrm{~dB}$ sound pressure level (SPL).
1. Install the sonometer microphone of the decibel $(\mathrm{dB})$ meter in the test chamber (the opening for installation is already bored by the manufacturer).

2. To reduce the background noise in the room, insulate the test chamber with a custom-built sound booth (see Figure 1B for an overview of the set-up).

3. Prepare a 96-well plate for the prepulse inhibition test. NOTE: The video camera has a $2048 \times 500$ pixel resolution, meaning only a maximum of 3 lanes (33 wells) can be imaged at a time.

1. Use a custom-made acrylic plate of 96-well format to reduce interference from shadows.

NOTE: The measurements for the custom plate can be found at the following website: https://zenodo.org/ record/3739378\#.XooyLW5uKas

4. With the aid of a transfer pipette, transfer $310 \mu \mathrm{L}$ of exposure solution/medium with one larva into each well.

5. Calibrate and measure the stimulus intensity using the volume knob of the stereo amplifier and a decibel meter respectively.

6. Register the maximum sound intensity in the "level reference" section.

\section{Stimulus parameters and video acquisition}

1. Turn on the computer, the amplifier system and the $\mathrm{dB}$ meter (see Figure 1A for an overview of set-up).

2. Use the volume knob by turning it to minimum or maximum to adjust the sound intensity.

1. Check the sound level with the $\mathrm{dB}$ meter each time, the volume knob is adjusted. This is important in finding the maximum and minimum sound intensity that can be produced by the set-up. 
NOTE: The dB meter computes the RMS dB output for the stimulus. The system generates the sound inside the solid components of the test-chamber, keeping the plate firm while producing a vibration in the horizontal plane of the entire plate support.

1. Adjust the volume knob to maximum, measure the sound intensity with the $\mathrm{dB}$ meter and use this value.

3. On the interface of the PPI generator, define the parameters: inter-stimulus interval represented as Delay; inter-trial interval represented as Acquisition delta time; duration of prepulse etc.

1. For prepulse alone trials, ensure that the "Amplitude" or Duration of stimulus for Startle parameters are set to zero and vice versa for startle alone trials.

4. To generate a trial list, select Add > give a name to the trial. For example, "Prepulse $50 \mathrm{~dB}$ alone".

NOTE: One can generate as many trials as desired, but be careful of how long the list is since this can crash the program.

1. Interleave prepulse trials with pulse alone trials in all PPI experiments using a pseudorandom order. Where multiple stimuli are presented in an experiment, an inter-trial interval (ITI) of $30 \mathrm{~s}$ is used.

NOTE: In this study, a 100 ms startle stimulus (pulse) of $660 \mathrm{~Hz}$, and $5 \mathrm{~ms}$ prepulse stimuli of $440 \mathrm{~Hz}$ were used. For PPI experiments, inter-stimulus interval (ISI) was $100 \mathrm{~ms}$.

5. To save the protocol, select File $>$ Save as.

6. Adjust lighting conditions in the test-chamber as follows.

1. Launch USB measurement computing, select analog out then go to D/A OUT O (P13) to make changes to the lighting. A value of zero means no light while increasing the D/A OUT O value, increases the intensity of light in the box. Light intensity of 100 was used for all experiments.

7. Set-up the camera

1. Launch the software and wait for the camera to load.

2. Select Adjustments (found on right-hand side) and set the acquisition frame rate to 1,000 then click apply to effect the change.

8. Acclimate larvae to a $100 \%$ light illuminated test chamber for 5 min before the experiments are started.

9. To begin an experiment, select the Experiment menu on the PPI generator, click Run and the select well format (e.g., 33 wells).

1. Always make sure that the camera software is launched with the right settings before running an experiment.

2. Acquire a $2 \mathrm{~s}$ video for each trial.

3. Make sure that the acquisition frame rate is set at 1,000 .

\section{Automated tracking and analysis of acoustic startle response and PPI}

1. Protocol setup.

1. Launch the analysis software (see the Table of Materials). Choose New from template > Apply a predefined template and then go through other menus (details below).

1. Choose from video file under Video Source.

2. Browse video file. Set subject as fish $>$ zebrafish larvae > zone template (no template). Specify Number of arenas under Arenas. 
3. Specify Number of subjects per arena (set as 1) under Subjects.

4. Select Center-point, nose-point and tail-base detection under Tracked Features (see Figure 2A,B).

NOTE: This is important to calculate the body angle of the C-start response (see Figure 2C).

5. Click Name > save as. Units used are mm, s, deg for distance, time and rotation respectively.

NOTE: Remember to use the same unit for calibrating the scale.

\section{Choose Arena setting.}

1. Click Grab background image.

2. Go through the steps on the right-hand menu (if in doubt, use the Help menu).

3. Choose the circle drawing tool to draw the arenas.

3. Choose Trial control settings $>$ create new $>$ name.

4. Choose Detection settings, go through the steps on the right-hand menu.

1. Set sample rate to 25. Choose advanced detection settings. Under Method, select dynamic subtraction, advanced model/adult fish, then set Subject color compared to background as Darker and move the slider to define the larva's contrast.

2. Under subject contour, select erode first, then dilate and increase the contour erosion and dilation values until the animal is completely detected.

5. Save the protocol and use for subsequent analyses of PPI videos acquired.
2. Trial list setup.

1. Choose trial list, define independent variables such as larval ID, treatment, stimulus type, etc. Select the path of videos and define a list of trials for batch acquisition.

3. Acquisition setup.

NOTE: If a trial list has been generated, one can perform a batch acquisition of the videos.

1. If some tracks are lost, use the track editor to adjust the tracked features.

2. Exclude from analysis, the tracking errors that remain unresolved after using the track editor.

3. Set track smoothing profile to $1 \mathrm{~mm}$ to decrease noise from data. This can be adjusted based on the background activity of larvae.

4. Analysis setup.

1. To select trials to be analyzed, choose Data profiles and define tracks based on the independent variable of interest.

NOTE: If components are hidden, click on the eye symbol to the upper right-hand corner to display.

1. Filter parts of trials to be analyzed (e.g., based on treatment or type of stimulus group).

2. Select part of the tracks to be analyzed (nesting). For this study, data was nested for tracks between onset of stimulus and 100 ms after stimulus onset.

3. Remember to connect all filters and nesting boxes with arrow lines to complete the instruction.

2. Define dependent variables to be analyzed, select Analysis profiles and specify the variables of interest (focus on Body under dependent variables). 
NOTE: If components are hidden, click on the eye symbol to the upper right-hand corner to display.

1. Double click Body angle. Select absolute bend. Go through Trial settings and select maximum, then click on add.

3. Double click body angle state. Set averaging interval to $\mathbf{5}$ samples. Set bend angle threshold. To calculate statistics for bent, go through Trial statistics and select latency to first $>$ add. Repeat steps until varying thresholds are obtained (between $20-80^{\circ}$ was used) and name accordingly.

4. Generating statistics and charts.

1. Choose analysis $>$ results $>$ statistics $\&$ charts, then click calculate.

2. Make sure the data and analysis profiles are set to the right template since several templates can be made under each section.

5. Export trial and group statistics as spreadsheet files for processing and analysis.

\section{Data analysis}

1. Open the spreadsheet file containing the trial statistics.

2. Select the columns Body angle Maximum deg, Bent latency (of the various body angle thresholds).

3. Consider every change in body angle $\geq 30^{\circ}$ within a cutoff latency of $50 \mathrm{~ms}$ after stimulus onset as a positive Cstart response (i.e., a responder); those with $<30^{\circ}$ body angle are non-responders.

4. In a binary fashion, assign 1 to a responding larva and 0 to non-responding larvae for each plate.

1. Count the total number of responders and nonresponder larvae for each plate. Calculate the responders $(\%)$ in each case calculated as (number of larvae responding/total number of larvae) $\times 100$. Exclude larvae that respond less than $30 \%$ to the startle stimulus from the analysis ${ }^{16}$.

NOTE: Any stimulus intensity capable of eliciting a Cstart response in equal to or more than $70 \%$ of the larvae is considered a suitable startle stimulus ${ }^{16}$.

5. Calculate \%PPI as $100 \times$ (percentage responding to startle stimulus - percentage responding to prepulse + startle sequence)/ (percentage responding to startle stimulus) ${ }^{16}$.

\section{Statistical analysis}

1. Present data as the mean \pm standard deviation, S.D. (see the Table of Materials for statistical software).

2. Determine the effects of varying prepulse intensities on larval response using one-way ANOVA followed by a Tukey's post-hoc test.

3. Use two-way ANOVA followed by Holm-Sidak's post-hoc test to determine the effects of drug treatment on \% PPI response with varying prepulse intensities.

\section{Representative Results}

Three experiments were performed to validate the protocol of combining multiple systems to analyze prepulse inhibition of acoustic startle response in the larval zebrafish. First, the ability to accurately deliver acoustic stimuli and to capture the response of larvae to the startle stimulus was tested. Next, was validating the ability to attenuate startle response when a prepulse stimulus is presented. Finally, the ability to detect the pharmacological modulation of prepulse inhibition of the startle response by the drugs apomorphine, haloperidol and ketamine was established. 


\section{Larval zebrafish response to acoustic startle stimuli}

Previous work has demonstrated that larval zebrafish display a characteristic $\mathrm{C}$-start when presented with startling acoustic stimulus $^{16}$. The ability to incite and capture the behavior of larvae to the startle stimuli was tested. Recorded larvae were observed to display the C-start response (Figure 2). A stimulus of $70 \mathrm{~dB}$ re $(100 \mathrm{~ms}, 660 \mathrm{~Hz}$, Supplementary Figure $1 \mathrm{~A}$ ) was strong enough to elicit response in $\geq 70 \%$ of the larvae (Figure 3A). When repeatedly presented 30 times at an inter-trial interval of $30 \mathrm{~s}$, the $70 \mathrm{~dB}$ re stimulus did not result in larval habituation ( $N=3$ replicates; 16 larvae/ replicate), as shown in Figure 3B.

\section{Prepulse decreases startle response of larval zebrafish}

to acoustic stimuli

A plethora of evidence shows that prepulse stimuli modulates larval response to a startle stimulus ${ }^{15,21,22,26}$. A twopulse paradigm was used, where a weak stimulus called the prepulse preceded the startle-inducing stimulus called the pulse. The prepulse stimuli used were either 20,17 , or $14 \mathrm{~dB}$ less than the pulse stimulus that was set at 70 $\mathrm{dB}$ re. The prepulse $(5 \mathrm{~ms}, 440 \mathrm{~Hz})$ was always presented $100 \mathrm{~ms}$ before the pulse onset (Supplementary Figure 1B). Each tested prepulse stimulus significantly reduced larval response to the pulse. In Figure 4 the larval response (in \%) to acoustic startle stimuli is shown for $6 \mathrm{dpf}$ TL in E3 medium, $N=6$ (16 larvae/group). The percentage of larvae responding to the startle stimulus (pulse) was $79.86 \pm 9.772$. Expectedly, when the startle stimulus was preceded by either a 50,53 or $56 \mathrm{~dB}$ prepulse, the larval response decreased to $40.87 \% \pm 11.30 \%, 39.58 \% \pm 7.345 \%$ and $29.17 \% \pm$ $9.350 \%$ respectively. One-way Anova analysis revealed a statistical difference in stimulus effect on larvae $(F(3,48)$ $=57.23, \mathrm{P}<0.0001)$ with Tukey's multiple comparisons test revealing statistical significance across groups at $95 \%$ confidence interval.

\section{Pharmacological modulation of prepulse inhibition}

Earlier studies showed that the dopaminergic drugs, apomorphine and haloperidol, as well as the glutamatergic drug, ketamine, significantly modulated prepulse inhibition in larvae just as in their mammal and rodent equivalents ${ }^{16}$. Concentrations for validation of the set-up were selected based on these studies. The inter-stimulus interval (ISI) for all the pharmacological experiments was $100 \mathrm{~ms}$.

\section{Effect of apomorphine on prepulse inhibition}

In Figure 5, larvae pretreated with $10 \mathrm{mg} / \mathrm{mL}$ apomorphine for 10 min displayed an overall reduction in \% PPI compared to E3 control larvae (two-way ANOVA, non RM (factors: treatment and prepulse intensities; treatment: $F(1,34)=$ 16.21, $p=0.0003$; prepulse intensity: $F(2,34)=8.674$, $P=0.0009$, this showed a non-significant interaction: $F(2$, $34)=2.514, p=0.0959)$. To investigate the differences in more detail, Holm-Sidak's post-hoc test revealed significant differences in the startle response between E3 control and apomorphine treated larvae at prepulse intensities $53(p=$ $0.0126)$ and $56(p=0.0044)$ but not at $50 \mathrm{~dB}(p=0.5813)$.

\section{Effect of haloperidol on prepulse inhibition}

Figure 6 shows an overall increase in \%PPI in larvae pretreated for $20 \mathrm{~min}$ with $20 \mu \mathrm{M}$ haloperidol compared to those in E3 medium (two-way ANOVA, non RM (factors: treatment and prepulse intensities; treatment: $F(1,32)=$ $20.75, p<0.0001$; prepulse intensity: $F(2,32)=3.147, p=$ 0.0565 , with no significant interaction: $F(2,32)=0.7455, p$ $=0.4826)$. Using the Holm-Sidak's post-hoc test, presence of statistical significance was observed only at prepulse 
intensities $53(p=0.00489$ and $56(p=0.0348)$ but not at 50 $d B(p=0.067)$.

\section{Effect of ketamine on prepulse inhibition}

Figure 7 shows that at different prepulse stimulus intensities, there were differences in the startle response between E3 control larvae and those pretreated for $10 \mathrm{~min}$ in $1.0 \mathrm{mM}$ ketamine (two-way ANOVA, non RM (factors: treatment and prepulse intensities; treatment: $F(1,35)=25.46, p<0.0001$; prepulse intensity: $F(2,35)=6.018, p=0.0057$, with no significant interaction: $F(2,35)=0.8450, p=0.4381)$. HolmSidak's post-hoc test, showed significance only at prepulse intensities of $50(p=0.0039)$ and $53(p=0.0027)$, but not at $56 \mathrm{~dB}(\mathrm{p}=0.0802)$.



B

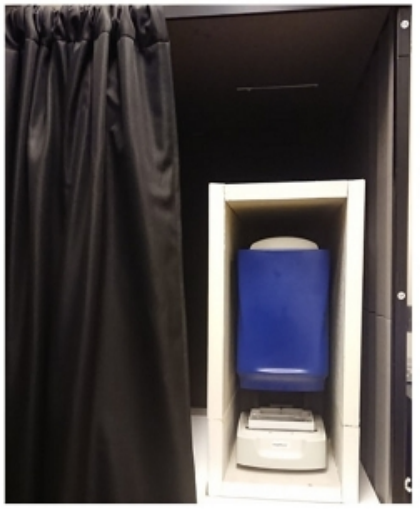

Figure 1: Testing apparatus. (A) Overview of equipment set-up. (B) In-house insulation of the set-up equipment to minimize background noise during experiments. Please click here to view a larger version of this figure. 

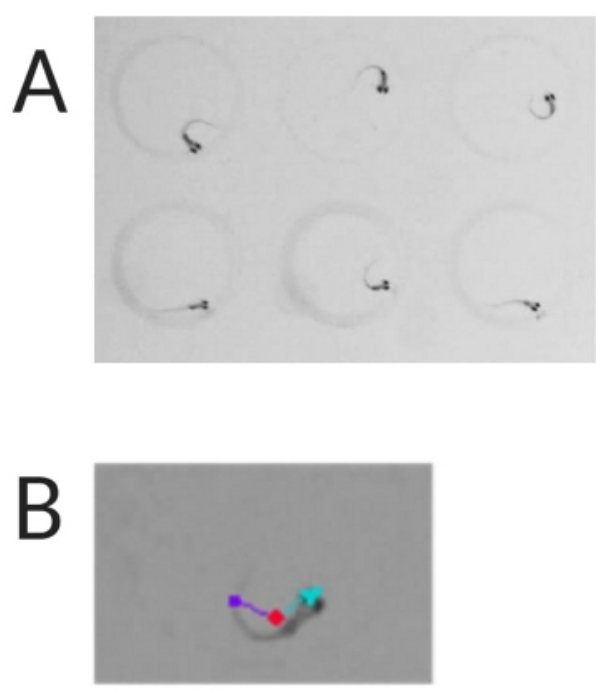

C

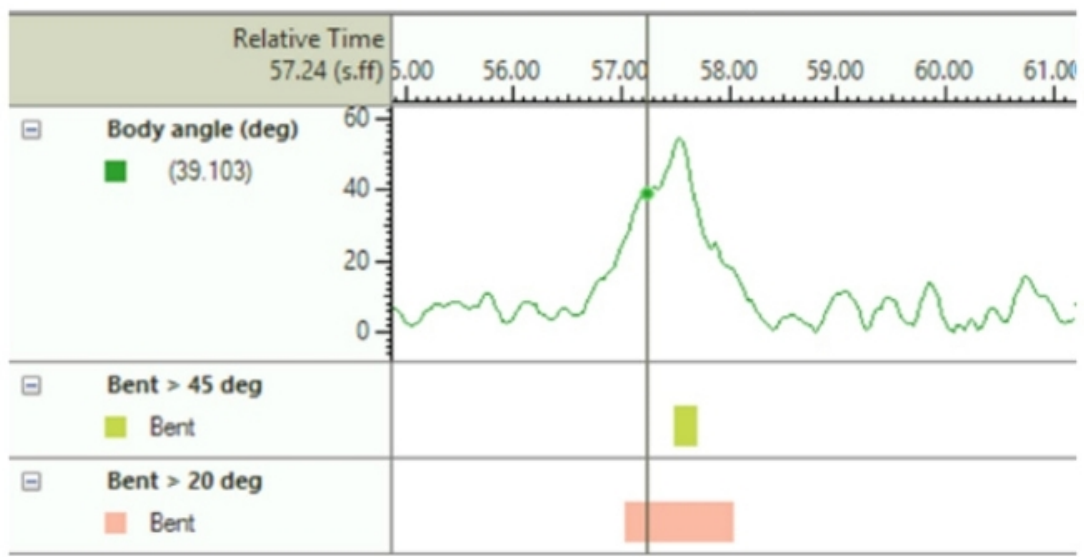

Figure 2: Analysis of the larval zebrafish acoustic startle response. (A) Characteristic C-start displayed by 6 zebrafish larvae at $6 \mathrm{dpf}$. (B) Representative image of the three tracked features superimposed on a $6 \mathrm{dpf}$ larva: center-point (red), nose-point (cyan) and tail-base (purple). (C) Representative image of the absolute bend angle displayed by a 6-dpf TL wild type larvae. Please click here to view a larger version of this figure. 



Figure 3: Determination of acoustic startle threshold. (A) A stimulus intensity of $70 \mathrm{~dB}$ (represented by red dash lines) is capable of eliciting a C-start response in $>70 \%$ of larvae $(\mathrm{N}=33 ; 6 \mathrm{dpf} \mathrm{TL}$ ). (B) Larvae do not habituate to $70 \mathrm{~dB}$ re stimulus presented 30 times (trials) at an inter-trial interval of $30 \mathrm{~s}(\mathrm{~N}=3$ replicates; 16 larvae/replicate). Data are presented as mean \pm S.D. Please click here to view a larger version of this figure. 


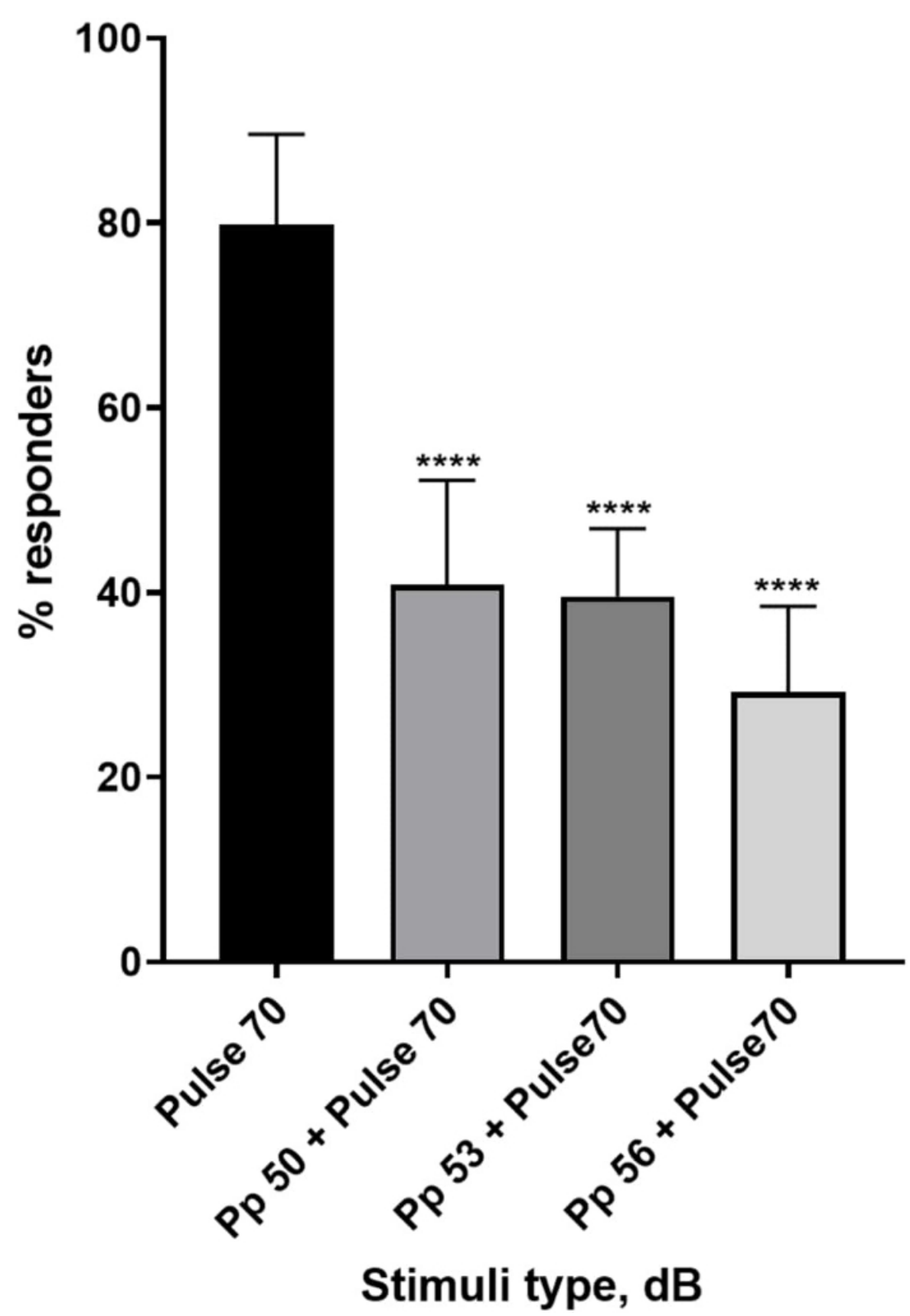

Figure 4: Pre-pulse-induced decrease in larval response (\%). Pre-pulse stimuli at 20, 17 and $14 \mathrm{~dB}$ lower than the $70 \mathrm{~dB}$ re startling stimulus cause a reduction in the number of wild type TL larvae C-start responders. All data represented as mean \pm S.D., $N=5$ (16 larvae/group), ${ }^{* * \star *} p<0.0001$, significantly different from startle stimulus by Tukey's post-hoc test after oneway ANOVA. Please click here to view a larger version of this figure. 


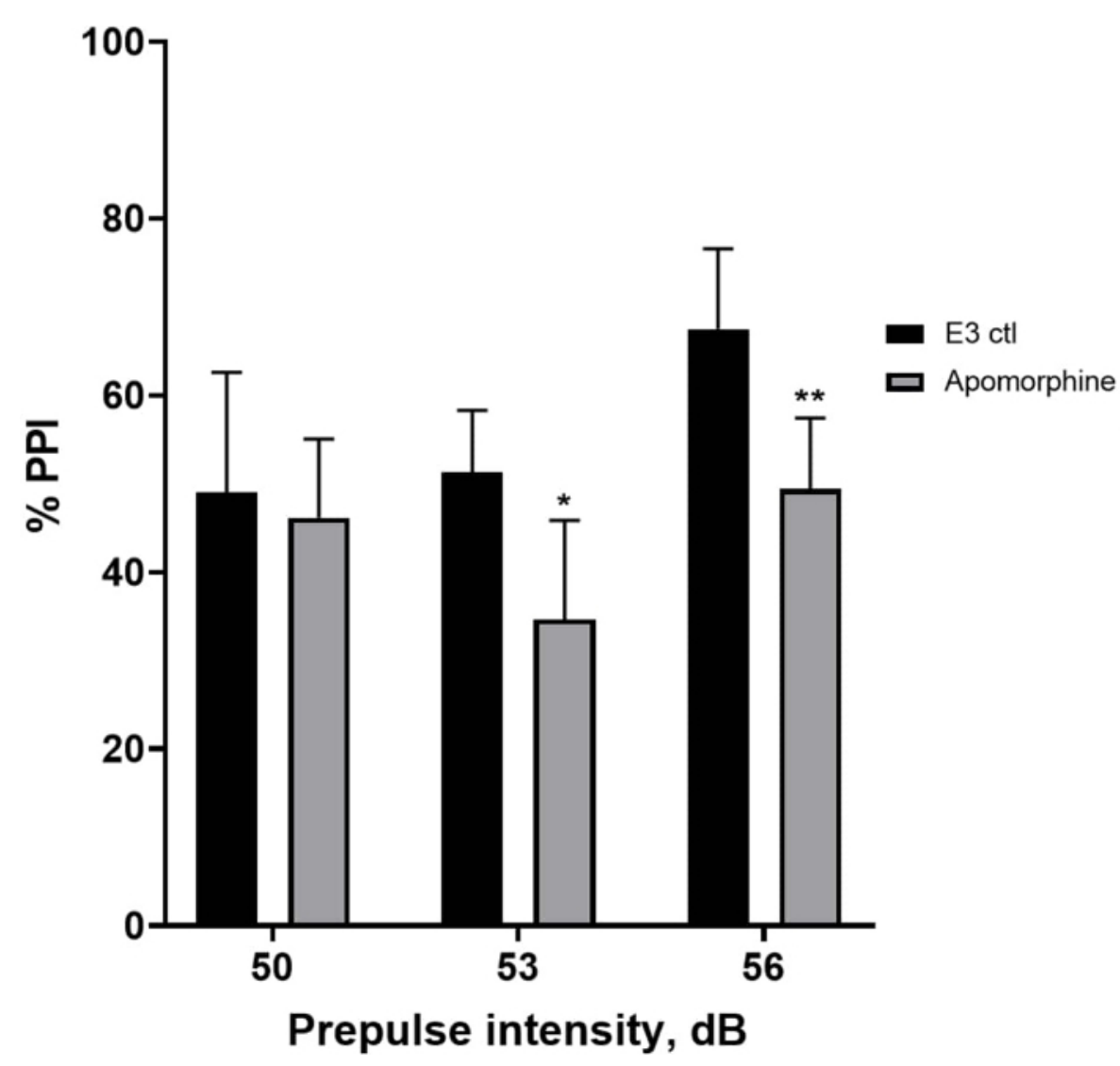

Figure 5: Apomorphine induced deficits in \%PPI. All data are presented as mean \pm S.D., N = 4-5 (16 larvae/group), statistically significant difference by Holm-Sidak's post-hoc test after two-way ANOVA. * $p=0.0126$, E3 ctl/apomorphine treated group at $53 \mathrm{~dB} ;{ }^{* *} \mathrm{p}=0.0044, \mathrm{E} 3 \mathrm{ctl} /$ apomorphine treated group at $56 \mathrm{~dB}$. Please click here to view a larger version of this figure. 


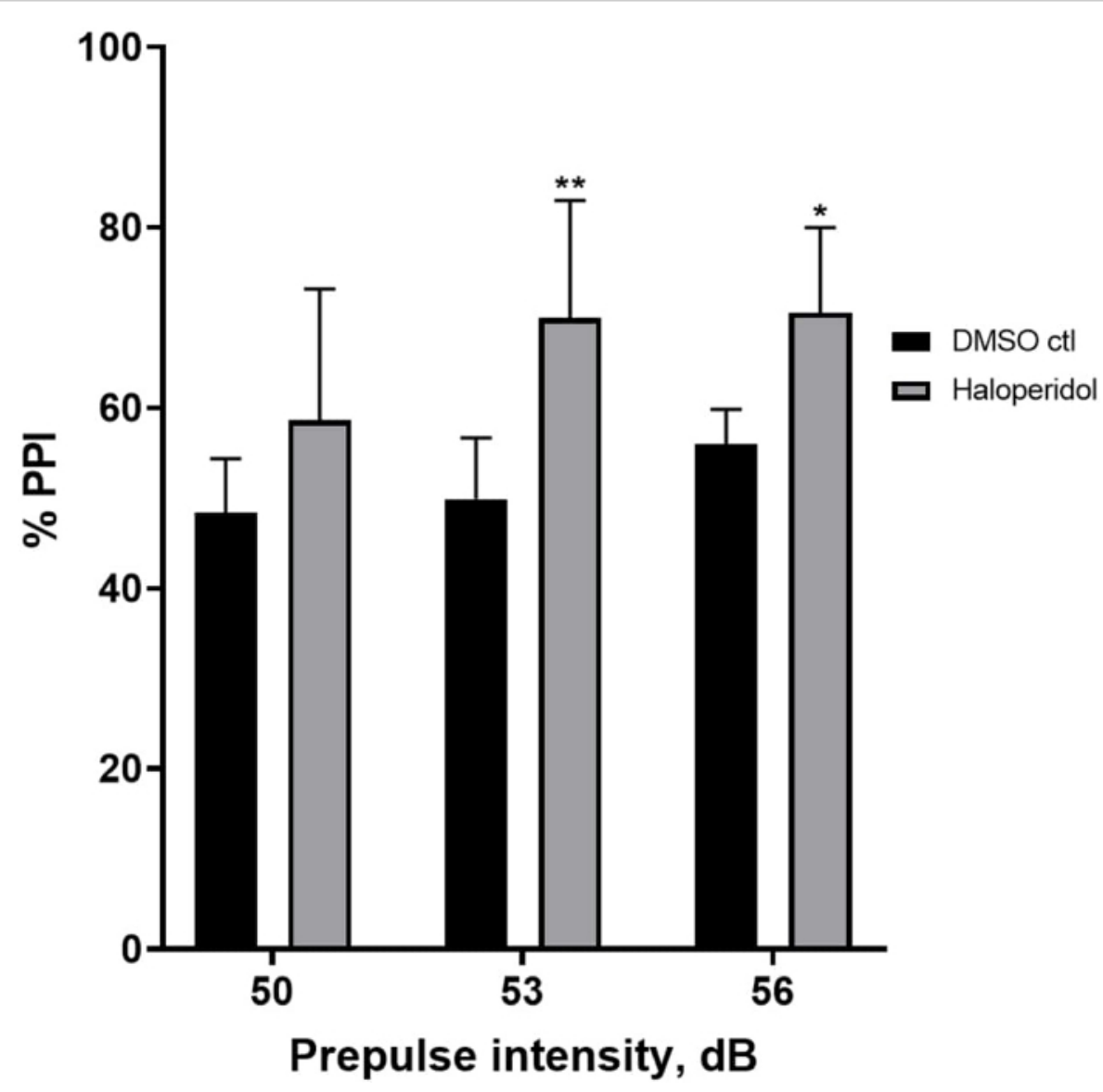

Figure 6: Haloperidol induced enhancement in \%PPI. All data are presented as mean \pm S.D., N = 4-5 (16 larvae/group), statistically significant difference by Holm-Sidak's post-hoc test after two-way Anova. ${ }^{* *} p=0.0048$, DMSO ctl/apomorphine treated group at $53 \mathrm{~dB} ;{ }^{*} \mathrm{p}=0.0348$, DMSO ctl/apomorphine treated group at $56 \mathrm{~dB}$. Please click here to view a larger version of this figure. 


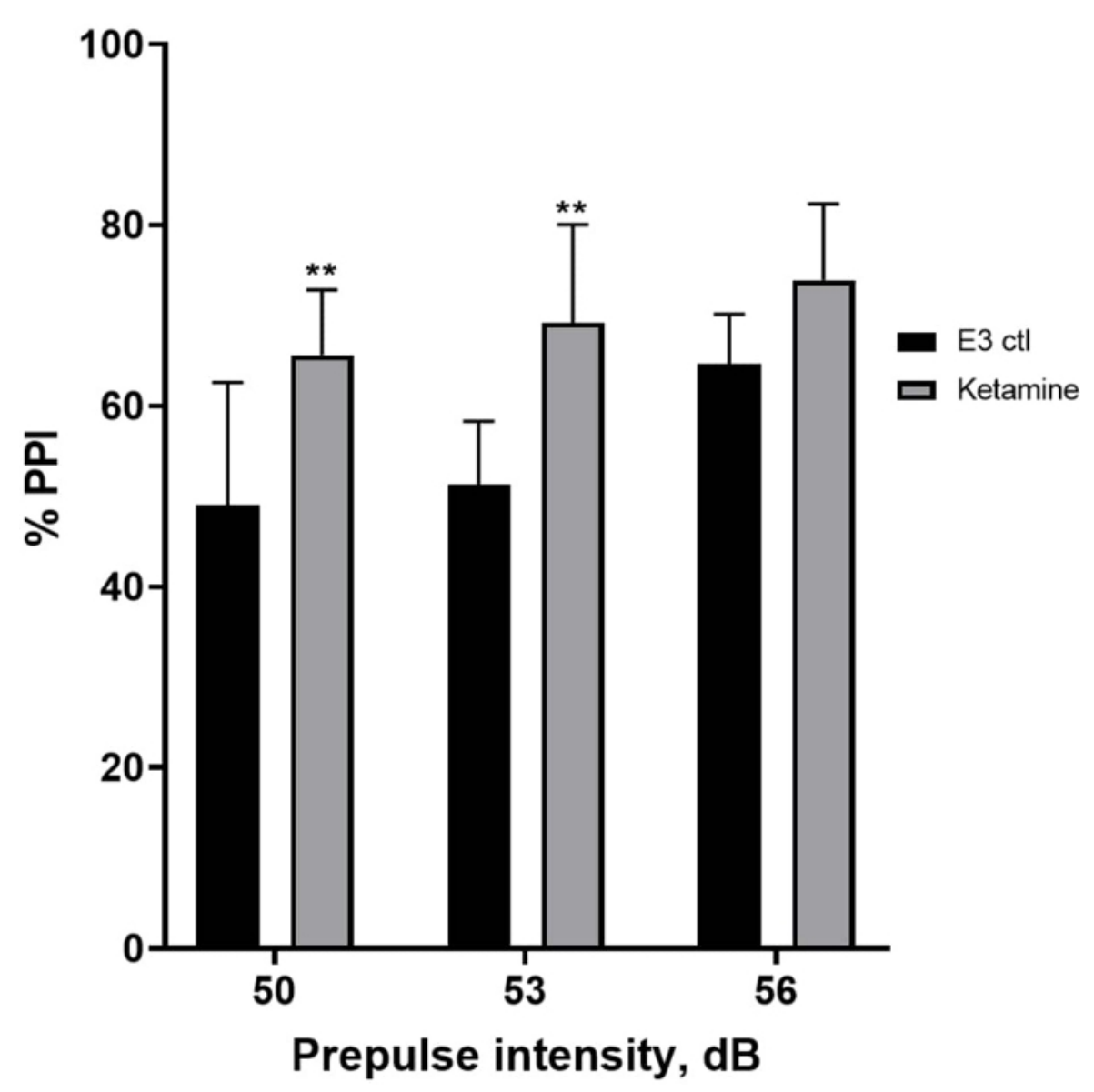

Figure 7: Ketamine induced enhancement in \%PPI. All data represented as mean \pm S.D, N = 4-5 (16 larvae/group), statistically significant difference by Holm-Sidak's post-hoc test after two-way Anova ${ }^{* *} p=0.0039$, E3 ctl/apomorphine treated group at $50 \mathrm{~dB},{ }^{* *} \mathrm{p}=0.0027, \mathrm{E} 3 \mathrm{ctl} /$ apomorphine treated group at $53 \mathrm{~dB}$. Please click here to view a larger version of this figure.

Supplementary Video 1: Representative video of larvae displaying a $\mathrm{C}$-start in response to $70 \mathrm{~dB}$ acoustic startle stimulus. Please click here to download this video.

\section{Supplementary Figure 1: Representative examples of} generated stimulus conditions using the PPI generator. (A) Stimulus alone trial, (B) pre-pulse inhibition trial (prepulse + pulse), (C) no stimulus trial to measure threshold baseline bend angle of unstimulated larvae. Please click here to download this figure.

\section{Discussion}

It is essential to validate any new behavioral assay system with the aim of improving and refining protocols for neurobehavioral research. In the current investigation, the ability of two commercially available systems and software to induce an acoustic startle response in zebrafish larvae and to detect and quantify previously described pharmacological modulation of such behaviors were assessed. 
A number of modifications and troubleshooting were performed to optimize the set-up. The default software for analysis of C-start responses was such that analysis automatically proceeded after the data for every experiment was acquired (22 trials/plate constituted an experiment). This reduced the number of plates that could be run per day, thus reducing the throughput (5 plates per day). To avoid this limitation, there was a need to de-couple the analysis software from the data collection process, which increased the throughput to an average of 10 plates per day. Thus, the decision to turn to an independent analysis software for nonlive analysis proved successful and more efficient. To avoid interference from shadows or other debris which introduces noise to the data, it is recommended to fill wells completely with medium, remove all bubbles and avoid food particles or similar which can be mistaken for larvae, thereby generating noise in the data. After calibration of the sound stimuli, the maximum intensity reachable by the amplifier system as captured by the $\mathrm{dB}$ meter was $85 \mathrm{~dB}$ re, while the initial background noise in the testing chamber was $60 \mathrm{~dB}$ re. This resulted in a narrow $\mathrm{dB}$ window in which to operate. Hence, it was critical to keep background noise as minimal as possible. To achieve this, parafon acoustics material (see Table of Materials) was used to build an additional layer of insulation around the test-chamber and an extra layer of insulation using a vocal booth bundle (see Table of Materials). With these layers of insulation, the background noise inside the testing chamber was successfully reduced from the initial $60 \mathrm{~dB}$ to $45 \mathrm{~dB}$ re.

Currently, one advantage of this set-up is that all the components are commercially available and as such, not limited to only a few labs. Individuals with limited knowledge in coding language can use it, as the protocol is rather easy to understand and follow. For example, by using the
PPI system, it was possible to deliver pulses and prepulses at varying inter-stimulus and inter-trial intervals, as well as capture larval responses to such stimuli. Once these behaviors were captured, they could be classified using the analysis software into responders and non-responders. The responder group was categorized as larvae that displayed a C-start of $30^{\circ}$ or more at a latency of $<50 \mathrm{~ms}$. In addition, the PPI response is modulated by drugs that target dopaminergic and glutamatergic signaling (reviewed by Geyer and colleagues ${ }^{27}$ ). Consistent with previous studies, apomorphine, a non-selective dopamine receptor agonist, reduced the pre-pulse inhibition of startle response in larval zebrafish, while haloperidol a dopamine antagonist enhanced the response. In larval zebrafish, ketamine has been shown to modulate PPI differentially based on the duration of the $|S|^{16}$. In the aforementioned study, larval PPI was enhanced at $30 \mathrm{~ms}$ but suppressed at $500 \mathrm{~ms}$ ISI when pre-treated with ketamine. Although this study did not use variable ISI, the observation that ketamine enhanced PPI at an ISI of $100 \mathrm{~ms}$, makes it comparable with the previous study's data when an ISI of $30 \mathrm{~ms}$ was used. The study demonstrated that by combining these commercially available systems, it is possible to perform the PPI assay and to reliably detect pharmacologically induced changes in the zebrafish larval PPI response. A limitation of the system is that the nosepoint feature tracked by the analysis software always falls on one of the eyes of the larvae, thereby creating a baseline angle. To overcome this, it is necessary to always determine the baseline bend angle of unstimulated larvae, which was found to be $\sim 30^{\circ}$ for larvae used in this study. Thus, forming the basis for the choice of $30^{\circ}$ as the threshold of what was considered a positive C-start response in startled larvae. If these points are taken into account, it should be possible to perform the PPI assay in any lab with access to the set-up equipment. This paper did not focus on categorizing 
the kinematics of startle response into short latency and long latency as reported earlier ${ }^{16}$, due to the scope of the variability of latency. Hence, only C-start responses $<50 \mathrm{~ms}$ after stimulus onset were used ${ }^{15}$.

Strain differences have been reported to influence zebrafish behavior in several assays $28,29,30,31$ as well as influence hearing sensitivity ${ }^{32}$. Hence, it is essential to determine the baseline bend angle of each strain tested. Since hearing sensitivities may also be different, it is crucial to determine baseline startle responses, the sound intensity most suited as either prepulse or startle stimulus for each strain and at what duration the stimulus is presented. The ISI is another parameter that should be carefully considered because some drugs can either enhance or reduce PPI based on the interval between the prepulse and startle stimulus onset ${ }^{16}$. The expectation is that, laboratories interested in studying cognitive function, neuropsychiatric disorders and hearing (auditory function) will find this PPI set-up and protocol useful in screening their pharmacological and/or genetic models. This protocol also provides a basis for high-throughput screening of compound libraries.

\section{Disclosures}

The authors declare no competing financial interests.

\section{Acknowledgments}

We thank Ana Tavara and João Paulo R. P. Santana for excellent fish care and invaluable help with testing and setting up of the soundproof booths, and Dr. Wietske van der Ent for initial support with setting up the EthoVision software. This study was funded by the Research Council of Norway (ISP, BIOTEK2021/ DigiBrain).

\section{References}

1. Gawel, K., Banono, N.S., Michalak, A., Esguerra, C.V. A critical review of zebrafish schizophrenia models: Time for validation? Neuroscience \& Biobehavioral Reviews. 107, 6-22 (2019).

2. Howe, $\mathrm{K}$. et al. The zebrafish reference genome sequence and its relationship to the human genome. Nature. 496 (7446), 498-503 (2013).

3. Kalueff, A.V., Stewart, A.M., Gerlai, R. Zebrafish as an emerging model for studying complex brain disorders. Trends in Pharmacological Sciences. 35 (2), 63-75 (2014).

4. Panula, P. et al. The comparative neuroanatomy and neurochemistry of zebrafish CNS systems of relevance to human neuropsychiatric diseases. Neurobiology of Disease. 40 (1), 46-57 (2010).

5. Kokel, D., Peterson, R.T. Chemobehavioural phenomics and behaviour-based psychiatric drug discovery in the zebrafish. Briefings in Functional Genomics and Proteomics. 7 (6), 483-490 (2008).

6. Basnet, R.M., Zizioli, D., Taweedet, S., Finazzi, D., Memo, M. Zebrafish Larvae as a Behavioral Model in Neuropharmacology. Biomedicines. 7 (1), 23 (2019).

7. Henry, J., Wlodkowic, D. Towards High-Throughput Chemobehavioural Phenomics in Neuropsychiatric Drug Discovery. Marine Drugs. 17 (6) (2019).

8. Behavioural measurement system | Laval zebrafish | Drosophila | Daphnia | insects | Products | Zantiks. at <https://zantiks.com/products/zantiks-mwp>. (2020).

9. Product List - ViewPoint. at <http://www.viewpoint.fr/en/ products>. (2020).

10. Observation chamber for zebrafish research DanioVision. Observation chamber for zebrafish research 
- DanioVision. at <https://www.noldus.com/daniovision/ observation-chamber>. (2020).

11. EthoVision XT - Video tracking software | Noldus. EthoVision XT - Video tracking software | Noldus. at <https://www.noldus.com/ethovision-xt>. (2020).

12. Gould, T.D., Gottesman, I.I. Psychiatric endophenotypes and the development of valid animal models. Genes, Brain and Behavior. 5 (2), 113-119 (2006).

13. van den Buuse, M. Modeling the Positive Symptoms of Schizophrenia in Genetically Modified Mice: Pharmacology and Methodology Aspects. Schizophrenia Bulletin. 36 (2), 246-270 (2010).

14. Acoustic startle modification as a tool for evaluating auditory function of the mouse: Progress, pitfalls, and potential. - Abstract - Europe PMC. at <https:// europepmc.org/article/PMC/5446932>. (2020).

15. Bhandiwad, A.A., Zeddies, D.G., Raible, D.W., Rubel, E.W., Sisneros, J.A. Auditory sensitivity of larval zebrafish (Danio rerio) measured using a behavioral prepulse inhibition assay. Journal of Experimental Biology. 216 (18), 3504-3513 (2013).

16. Burgess, H.A., Granato, M. Sensorimotor Gating in Larval Zebrafish. Journal of Neuroscience. 27 (18), 4984-4994 (2007).

17. Eaton, R.C., Farley, R.D., Kimmel, C.B., Schabtach, E. Functional development in the mauthner cell system of embryos and larvae of the zebra fish. Journal of Neurobiology. 8 (2), 151-172 (1977).

18. SR-LAB - San Diego Instruments Startle Response. San Diego Instruments. at <https://sandiegoinstruments.com/ product/sr-lab-startle-response/>. (2020).
19. Startle packages - Med Associates Inc. Med Associates Inc. at <https://www.med-associates.com/productcategory/acoustic-startle-reflex-packages/>. (2020).

20. Startle response \& Pre-pulse inhibition test | O'HARA \& CO.,LTD. at <https://ohara-time.co.jp/products/startleresponse-pre-pulse-inhibition-test/>. (2020).

21. Thyme, S.B. et al. Phenotypic Landscape of Schizophrenia-Associated Genes Defines Candidates and Their Shared Functions. Cell. 177 (2), 478-491.e20 (2019).

22. Privat, M. et al. Sensorimotor Transformations in the Zebrafish Auditory System. Current Biology. 29 (23), 4010-4023.e4 (2019).

23. Gerlai, R. Reproducibility and replicability in zebrafish behavioral neuroscience research. Pharmacology Biochemistry and Behavior. 178, 30-38 (2019).

24. Add-on - Fast camera 1000 FPS - ViewPoint. at <http://www.viewpoint.fr/app.php/en/p/equipment/addon-fast-camera>. (2020).

25. Aleström, P. et al. Zebrafish: Housing and husbandry recommendations: Laboratory Animals. (2019).

26. Bhandiwad, A.A., Sisneros, J.A. Revisiting Psychoacoustic Methods for the Assessment of Fish Hearing. Fish Hearing and Bioacoustics: An Anthology in Honor of Arthur N. Popper and Richard R. Fay. 157-184 (2016)

27. Geyer, M.A., Krebs-Thomson, K., Braff, D.L., Swerdlow, N.R. Pharmacological studies of prepulse inhibition models of sensorimotor gating deficits in schizophrenia: a decade in review. Psychopharmacology. 156 (2-3), 117-154 (2001). 
28. Lange, M. et al. Inter-Individual and Inter-Strain Variations in Zebrafish Locomotor Ontogeny. PLOS ONE. 8 (8), e70172 (2013).

29. Liu, X. et al. Strain-dependent differential behavioral responses of zebrafish larvae to acute MK-801 treatment. Pharmacology Biochemistry and Behavior. 127, 82-89 (2014).

30. Loucks, E., Carvan, M.J. Strain-dependent effects of developmental ethanol exposure in zebrafish. Neurotoxicology and Teratology. 26 (6), 745-755 (2004).

31. Bos, R. van den et al. Further characterisation of differences between $T L$ and $A B$ zebrafish (Danio rerio): Gene expression, physiology and behaviour at day 5 of the larval stage. PLOS ONE. 12 (4), e0175420 (2017).

32. Monroe, J.D. et al. Hearing sensitivity differs between zebrafish lines used in auditory research. Hearing research. 341, 220-231 (2016). 